\section{THE RADICAL TREATMENT OF CHRONIC INTESTINAL TUBERCULOSIS, WITH SUGGESTIONS FOR TREATMENT IN MORE ACUTE DISEASE AND IN TUBERCULOUS PERITONITIS. ${ }^{1}$}

BY A. W. MAYO ROBSON, F.R.C.S. ENG., VICE-PRESIDENT, ROYAL COLLEGE OF SURGEONS OF ENGLAND.

THE following cases of chronic tuberculous disease in the antestine treated surgically have raised hopes in my mind as to the possibility of doing good not only in chronic cases such as are here related but in some more acute cases where if the disease be strictly limited a radical operation might remove it, but where if more extensive a short-circuiting might set the part at rest and relieve it from irritation, thus renabling the ulcers to heal ; the surgical measures being supplemented by general, medical, and hygienic treatment. Whether these hopes will be realised or not I trust that the cases in themselves may prove of sufficient interest to warrant $m e$ in bringing them to notice. I think my readers will agree that whatever may be their views on the treatment of acute intestinal ulceration, in chronic intestinal tuberculosis, especially if associated with stricture, surgical treatment is advisable.

CASE 1. Tuberculous disease of the appendix extending to the cooum and ileum; stricture of the ileo-cacal valve; removal of the coeum and adjouning part of the ileum and ascending colon. - A man, aged 23 years, was sent to me on June 19th, 1900, with the history that after symptoms pointing to chronic appendicitis an operation had been undertaken by an experienced surgeon who found tuberculous disease of the appendix without any general affection of the peritoneum or glands, but as there were numerous adhesions and some matting of viscera in the neighbourhood the appendix was not removed and the abdomen was closed. The patient was in co way relieved and steadily grew worse generally and locally. When I saw him be was very thin and gave the history of having had pains in the cæcal region for 18 months, associated at times with paroxysmal pain extending across the abdomen, the paroxysmal attacks having recently become very frequent. There had been great loss of flesh with night sweats and diarrhcea. A hard swelling could be felt in the creal region and a coil of intestine below the umbilicus could be felt to contract and to harden under the examining hand, indicating chronic obstruction.

Operatiou was performed on July 17th, 1900. An oblique incision along the outer border of the right rectus was made. There was miliary tubercle on the peritoneum with slight serous effusion, also considerable matting of the viscera adjacent to the cæcum. The tumour, consisting of the crum, the lower third of the ascending colon, the appendix, and several inches of ileum, was removed, and the open end of the ileum was connected to the ascending colon by means of continuous sutures applied over a decalcified bone bubbin. on account of adhesions it was difficult to explore the rest of the abdomen, but no other mass could be discovered and the mesenteric glands, except those in the vicinity of the lower end of the ileum, were apparently not involved. The wound healed by first intention and the sutures were removed on the seventh day, but on the tenth day a small purulent collection was evacuated from the lower end of the scar and the length of time that the slight sinus took to heal suggested tuberculous infection. He returned home on August 29th markedly improved in his general health. Fresh air and general tonic treatment were advised. On June 3rd, 1901, nearly a year later, the medical superintendent of a Durham sanatorium wrote to me to say that the patient was improving generally but that he had had some abdominal pain suggestive of obstruction by a constricting band. A further exploration to ascertain if there are any bands or other removeable cause of obstruction has not yet been pressed as it has not appeared to be absolutely necessary and the general health has improved.

The mass removed showed tuberculous ulceration with 1902

A paper read before the Clinical Society of London on Jan. 10th, great thickening of the cæcum and ileum, the disease having apparently started in the appendix and then extended to the cæcum and involved the ileo-cæcal valve, which would barely admit the tip of the little finger.

CASE 2. Multiple tuberculous strictures of the ileum; enterectomy. - For the history of the following cases I am indebted to my dresser, Dr. J. Acomb. The patient was a woman, aged 51 years, who was seen with Dr. R. Petch of York. With regard to the family history, on the father's side it was negative but on the mother's side it was decidedly tuberculous. As to her previous history, she appeared to overgrow her strength between the ages of 10 and 14 years. When 10 years old she had a glandular swelling in the neck which was incised. She had no cough until 12 or 13 years ago, since which time she had been troubled by bronchial catarrh every winter. There was no perspiration at night. She had recently lost flesh and strength very considerably. For some 15 to 20 years the patient had suffered from so-called "bilious" attacks accompanied by vomiting and pain across the abdomen which would pass away after a few hours with diarrhoea. She was of a bad colour at the time of the attacks but had no apparent jaundice. These attacks occurred for some years, with perhaps a month's interval between each. Five years ago the character of the attacks appeared gradually to change and three years ago their place was taken by a constant pain across the waist and back. The pain did not radiate and was accompanied by indigestion and flatulence. She was subject to attacks of constipation for two or three days, followed by diarrhoea for three or four days, which would be accompanied by griping pain and would come on quite suddenly and unexpectedly. No distension was nuted; there was no tenderness during the intervals of pain and no tenesmus; the stools, as a rule, were watery. There was slight bleeding from the bowel on defecation five or six years ago but no blood had been noticed since. When seen by me on July 22nd, 1901, the patient was very thin and it was easy to see vermicular contraction of the intestines, the tumour coming and going at varying intervals, the pain being at times very severe. On examining by the rectum a nodular growth could be felt in Douglas's pouch, which was quite moveable from side to side and could be pushed out of reach. Operation was advised and was performed in York on July 26th.

The abdomen was opened in the middle line and the tumour previously felt by the rectum was discovered to be a coil of intestine very much thickened and forming a mass, as shown in the illustration (Fig. 1), there being no less

FIG. 1.

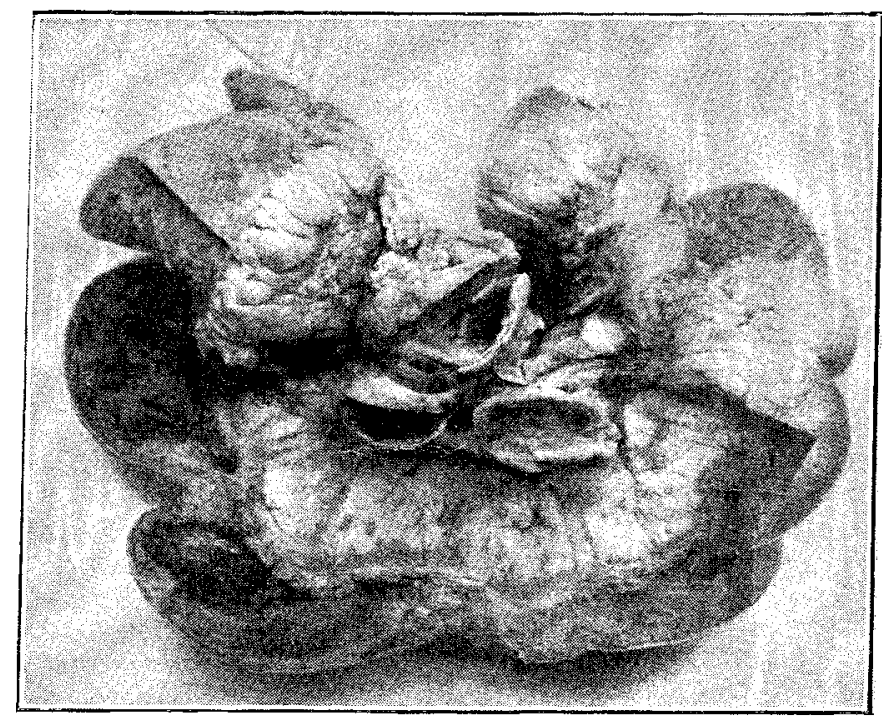

Tuberculous disease of the ileum with multiple strictures and enlarged glands.

than four distinct strictures, the first within one and a half feet of the last and all in the ileum. The affected area was brought through the incision, surrounded by sterilised gauze and excised, the healthy bowel being sutured end to end over a decalcified bone bobbin.

I have not seen the patient since but have beard that she recovered from the operation and gained fesh and strength. 
A year later she was well in every respect. The specimen removed was examined by Dr. Norman Smith, pathologist to the Leeds Infirmary, who gave the following report: "There is a great increase of small round leucocytes and of connective tissue in the submucous layer. At places the leucocytes form den e, deeply-stained aggregations. Seen by the higher powers they are made up of peripheral parts composed of small cells and a central portion of large cells. They are apparently giant-cell arrangements. At piaces in the muscular coat the same arrangement is commencing to be formed." The diagnosis was chronic tuberculosis of the bowel. The specimen is an extremely interesting one as showing how near to stenosis it is possible to get without complete obstruction; it also shows a gall-stone entangled in a pouch between two of the strictures.

CASE 3. Multiple tuberculous strictures or the ileum; short-circuiting. - The patient was a woman, aged 20 years, whom I saw with Dr. J. G. E Colby of Malton on March 6th, 1901. She gave the history that she had always been weak and thin since the age of 10 years but this was accounted for by her having grown so rapidly. At the age of $15 \frac{1}{2}$ years she began to have pain in the abdomen on the rignt side associated with diarrhoea two or three times a day. From that time onward she had never been long free from pain and diarrhoea and lately her attacks were coming on with greater frequency. Her loss of flesh and weakness was very marked and she suffered from night perspirations. No tumour had been discovered and she had not been troubled with vomiting. The motjons contained neither blood nor slime, but were, as a rule, quite fluid, like water. The pain came on directly after food for five minutes, then passed away, and two hours later diarrhœa usually followed. On examining the abdomen there were a rigid right rectus and tenderness in the cæcal region, but no swelling could be felt. Stricture was diagnosed and operation was advised. Although she tried fully various forms of medical treatment with careful dieting and plenty of fresh air there was no improvement and on June 10th I operated on her at a surgical home in Leeds. The ileum was found to be strictured in several places and the bowel generally was thickened over a distance of two feet before its termination in the crcum. Miliary tubercles were scattered over the affected bowel and the adjoining mesentery, but the abdomen was not generally invaded by tubercle. There was some free fluid in the peritoneal cavity. As the patient was so very feeble it was thought that she would not survive the removal of so much as two feet of the bowel; shortcircuiting was therefore performed, the ileum being connected to the transverse colon by means of a decalcified bone bobbin. The wound healed by first intention and the patient was sent home to the country on July 4th, and a very good report has been received of her progress. It was intended that she should return in order to have the diseased bowel excised, but her gain in health and the relief from the diarrhoea have made her shy of facing any further operation which I think may not be necessary. She is now said to be in good health.

CASE 4. Stricture or the duodenom probably tuberoulous, associated with tubercle of the stomach and stenosis of the pylorus.-The patient, a feeble girl, aged 19 years, was seen in 1895. She only weighed 4 stones 10 pounds and gave a history of vomiting and pain after food for four years though the loss of flesh had only been very pronounced during six months. She was deathly pale and was so thin that the skin was tightly stretched over the bones. Tuberculous glands were felt in the neck. The abdomen was swollen. The stomach was markedly dilated. On opening the abdomen the pylorus was found to be thickened and covered with tuberculous nodules which were scattered over the omentum and other viscera. Some free fluid was found in the peritoneal cavity. On laying open the pylorus to perform pyloroplasty the finger was passed into the duodenum and encountered a stricture one inch beyond the pyloric ring; this was evidently the result of ulceration. The tip of the little finger could just pass through it. A Weiss dilator was employed to stretch the stricture which was dilated so as to allow the thumb to pass readily through it. The pyloric incision was then closed by sutures transversely to the axis of the canal. For a week the after-operative progress was satisfactory, the wound healed and the stitches were removed, but no strength was gained and the gradually failed and died from exhaustion in the second week. With my present experience I should have performed gastro-enterostomy and begun feeding at once and I believe the result would have been different.
CASE 5. Tuberoulosis of the aceonm; entereotony.-(This report was furnished by Mr. J. F. Dobson, resident surgical officer.) The patient, a woman, aged 22 years, was admitted to the Leeds Infirmary on Nov. 15th, 1897. The patient had suffered from dyspepsia for several years. About six months previously she noticed that her side was painful when she turned and that she could not bear the pressure of her stays. This became worse and was accompanied by attacks of rather acute abdominal pain, principally in the right side. She never vomited during the attacks and had never been jaundiced. The pain had no relation to meals. The bowels were regular, generally rather loose ; no blood or mucus was noticed. The appetite was poor and there had been con siderable wasting of late. Menstruation commenced at 17 years of age; lately it had been scanty and painful. The tumour was felt in the region of the cecum but tenderness was not marked. At the operation the tumour was found to involve the cæcum and the commencement of the ascending colon. The lumen of the gut was not occluded but narrowed. No general tuberculosis of the peritoneum was observed and no enlaj gement of the glands was found. The tumour was excised and the ileum and ascending colon were united by continuous sutures over a decalcified bone bobbin. The wound healed kindly and the vatient improved rapidly and was discharged in fair health on Jan. 8th, 1898. Although the health was regained and there was no further return of the abdominal trouble the patient had pleurisy some months afterwards and is said to have died nearly a year later at her home. The pathologist's report was that a portion of tissue removed from the wall of the cæcum was of a pale grey colour, fairly elastic and translucent. Microscopically, delicate fibrous tissue with a few round and spindle cells between was seen. Many rounded and oval areas occurred where collections of round cells were tightly packed. In the midst of each group one or more well-developed giant cells were found. Appended is an illustration of the specimen removed at the operation (Fig. 2).

\section{Fig. 2}

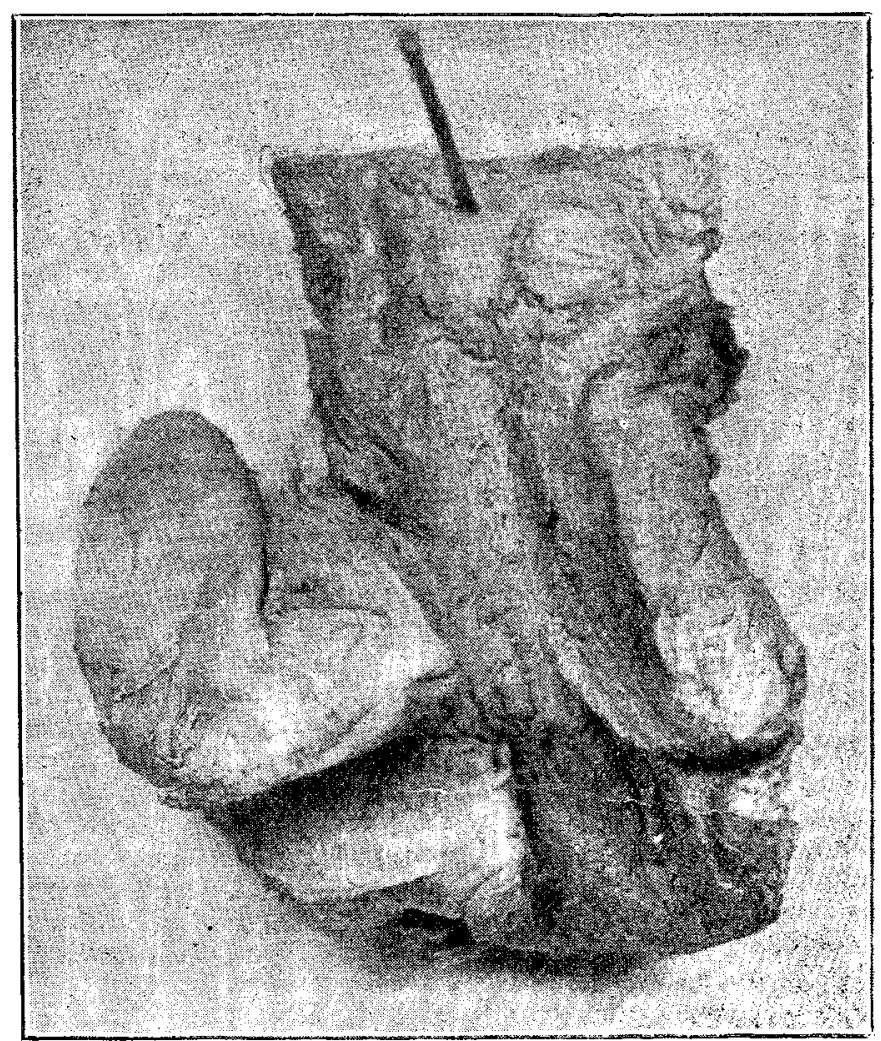

Tuberculous disease of the cæcum.

CASE 6. Tuberculons disease of the reotum and sigmoid flexure of the colon; colotomy and partial colectomy. - A man, aged 37 years, was admitted to the Leeds Infirmary on Oct. 31st, 1898, suffering from a profuse purulent discharge from the anus associated with great pain, which increased on defecation, and with diarrhcea and great deterioration of health. He gave the history of having had an operation for "fistula in ano" three years before, from which he 
recovered; but six months later he noticed the motions to be streaked with blood and slime which gradually increased in quantity until it began to come independently of normal bowel action. On examination by means of a speculum the whole of the rectum was found to be involved in ulceration, which extended upwards beyond reach of the proctoscope. The sigmoid flexure could be felt to be much thickened and was tender to pressure. Inguinal colotomy was performed, the sigmoid being drawn out as far as possible in order to secure healthy bowel being opened; the diseased part external to the abdominal wall was then cut away. This was examined by the resident pathologist and proved to be tuberculous. After the first week the lower bowel was regularly syringed out with the boric acid solution containing a tablespoonful of tincture of hamamelis to the pint, the stream being forced alternately from the anus to the colotomy opening and vice versî. Convalescence was very slow, but he steadily picked up strength and after going to the convalescent home was discharged much relieved.

Remarks.-Apart from cancer, stricture of the bowel usually results from ulceration with consecutive changes in the coats of the bowel, but there is a specimen in the Leeds School Museum which shows a stricture due to gradual invasion of the bowel walls by tubercle spreading from glands in the mesentery, and I have also seen, and operated on, a case of. stenosis produced by fibrous hyperplasia extending from a mesenteric tuberculous gland in process of cure. In the first four cases here related there was no serious difficulty in coming to the conclusion that stricture wa- present and from the length of time that the obstructive symptoms, constipation alternating with diarrhœa and pain, had existed that the narrowing was simple in character and not malignant. In three of the cases the family and personal history, the early age, the loss of flesh, and night perspirations were suggestive cf the stricture being tuberculous; moreover, there was an absence of history of typhoid fever or dysentery, the two other chief causes of intestinal alceration, and of hernia or injury which sometimes leads to intestinal stenosis. In Case 5 it was suspected that the tumour might be malignant and, in fact, it was only on careful examination subsequently to removal that its true character was discovered. The cases related afford examples of tuberculous ulceration in parts of the intestinal tract from the duodenum to the anus, though the most common site of the disease is in the lower ileum and about the ileo-cæcal valve.

Chronic tuberculous ulceration leading to stricture is evidently not very common, though perhaps some cases put down as malignant, but where cure occurs after colotomy or enterectomy, may be of this nature. I have in my mind two cases of apparent cure of malignant stricture by colotomy, one in a man, aged 33 years, in which I subsequently closed the colotomy opening after the health of the patient had become fully re-established and when there was manifestly no further need for the artificial anus; and another, in a man, aged 58 years, where the tumour exposed at the time of the colotomy has apparently disappeared and the patient is anxious to have the artificial anus closed.

Now, although both of these cases had all the appearances and feel of carcinoma, I think the subsequent course of events makes it extremely probable that the intestinal thickening leading to stenosis was tuberculous, though proof is wanting. The extension of the tuberculous process from the the appendix in Case 1 is apparently not a common condition, though seeing that the appendix is composed largely of lymphoid tissue it makes one wonder that it should comparatively rarely be attacked by tubercle, especially when the inflammatory attack is repeated over and over again. I have, however, operated on at least five cases of tuberculous appendicitis, and the removal of the diseased part has issued in recovery and gain of health. The following is a good example.

OASE 7. Tuberculous appendicitis; appendiceotomy.-A youth, aged 17 years, was sent to me on Oct. 1st, 1901, from the west coast, in such a weak condition that it was feared he could hardly bear any operation; he was suffering from night sweats and had a hectic temperature. There was well-marked tenderness over the cæcum with rigidity of the muscles. The history given was that he had had acute appendicitis 18 months before and that the abscess had been drained but the appendix had not been removed. A recurrence of the attacks with deterioration of health led to two attempts at removal of the cause, but the appendix, it was said, could not be found. At the operation on Oct. 6th there was well-marked tuberculosis of the croum with miliary tubercles scattered in the neighbourhood, though the rest of the peritoneum was free. After carefully detaching numerous and firm adhesions, the appendix, which was tuberculous, was found in the centre of offensive thick caseous pus, behind the cæcum and ascending colon. After removal of the appendix, clearing out and purifying the puscontaining cavity, the abdomen was closed, a single gauze drain being used. Recovery was uninterrupted and he returned home at the end of the month. A report was received from Mr. C. H. S. Vinter in December to say that the patient was well and getting out as usual.

The stricture in Case 1 was caused by a stenosis of the ileo-cæcal valve, but the lumen of the adjacent ileum and cæcum was also seriously narrowed by thickening of their coats. Cases 2 and 3 are characteristic examples of tuberculous ulcers, which usually begin in the lymphoid follicles and adopt a transverse direction, following the course of the vessels around the whole circumference of the bowel, thus leading to stricture in chronic cases from the lymph, which is thrown out to limit the ulcerative process, tending to contract. The situation in the ileum and the multiple characters of the strictures were also characteristic of tubercle. While in Cases 1 and 7 the adhesions to adjoining parts were most intimate in the other cases the affected part of the bowel was quite free, thus rendering operative measures quite easy and simple. In Cases 1, 2, 3, and $5 \mathrm{my}$ decalcified bone bobbin was employed as a temporary splint over which to apply sutures, a method which I have described on numerous occasions and which I still employ and advocate as being simple and efficient. The physiological rest to the diseased parts secured by short-circuiting as in Case 3, and by colotomy as in Case 6, seemed to answer so well that where the radical operation of excision would involve serious difficulties or the patient is not in a condition to bear it I would not hesitate in future to be content with similar procedures and then to trust to general treatment and hygienic surroundings to assist in the cure. If in the future my suggestion as to operative treatment in acute cases be thought advisable I anticipate that short-circuiting the diseased area will be found to be the more useful procedure, as it is the easier and safer method to pursue; but expericnce only can show whether these suggestions will prove of so much service in acute as they are in the chronic cases.

It is interesting to note that in Cases 1 and 7 where there were tuberculous nodules in the peritoneum-where, in fact, there was tuberculous peritonitis co-existent vith the bowel disease-the mere exploratory operation had not cured or even arrested the tuberculous process, but that the radical operation of removing the principal focus of disease, or, as in Case 3, the short-circuiting and so setting at rest the diseased bowel, was in all three cases effectual in putting a stop to the tuberculous peritonitis and, so far as one can say without seeing the parts exposed, in curing the more diffuse disease.

Now all surgeons must have had the experience of curing tuberculous peritonitis by simple abdominal section with or without drainage and probably many of us have also experienced disappointment in other cases where the effusion has returned after a longer or shorter interval. May the explanation of these recurrent cases lie in the fact that the original focus of disease in the ovaries, Fallopian tubes, or bowel has not been removed and has been again the startingpoint for a further diffusion of the tubercle throughout the peritoneal cavity? If this be a correct surmise the remedy is quite clear, that wherever the original disease may be it should be removed if that be possible without adding seriously to the risk of operation. I have acted on this principle in a number of operations for tuberculous peritonitis and have removed masses of tuberculous, mesenteric, or retro-peritoneal lymphatic glands, tuberculous Fallopian tubes and ovaries, loops of tuberculous intestine, and tuberculous appendices, and this with such success as to encourage me in following up the method and in recommending it to the attention of other surgeons. Leeds.

Public Health of Gramorganshire.-Dr. W. Williams, the medical officer of health of the county of Glamorgan, in his annual report for 1901 , which has just been issued, states that during the year 22,173 births and 11,747 deaths were registered respectively; 604 deaths were attributed to pulmonary tuberculosis. 